\title{
The Cerebellum in Emotion Regulation: A Repetitive Transcranial Magnetic Stimulation Study
}

\author{
Dennis J. L. G. Schutter • Jack van Honk
}

Published online: 15 October 2008

(C) The Author(s) 2008. This article is published with open access at Springerlink.com

\begin{abstract}
Several lines of evidence suggest that the cerebellum may play a role in the regulation of emotion. The aim of this study was to investigate the hypothesis that inhibition of cerebellar function using slow repetitive transcranial magnetic stimulation (rTMS) would lead to increased negative mood as a result of impaired emotion regulation. In a randomized counterbalanced within-subjects design, 12 healthy young right-handed volunteers received 20 min of cerebellar, occipital, or sham $1 \mathrm{~Hz}$ rTMS on three separate days. Mood state inventories were acquired prior to and immediately after rTMS and after an emotion regulation task (ERT). In the ERT, participants were instructed to either look at aversive and neutral scenes, or to suppress the negative feelings experienced while watching aversive scenes during which the electroencephalogram (EEG) was recorded. Results showing no changes in baseline-corrected mood were observed immediately after rTMS. However, significant increases in baseline-corrected negative mood following the ERT were reported after cerebellar rTMS exclusively. No effects on the EEG during the ERT were observed. These findings provide support for the view that the cerebellum is implicated in the regulation of emotion and mood, and concur with evidence of cerebellar abnormalities observed in disorders associated with emotion dysregulation. In order to clarify the underlying biological mechanisms involved, more research is needed.
\end{abstract}

Keywords Cerebellum - Electroencephalogram . Emotion regulation - Transcranial magnetic stimulation

D. J. L. G. Schutter $(\bowtie) \cdot$ J. van Honk

Experimental Psychology, Helmholtz Research Institute, Utrecht University,

Heidelberglaan 2,

3584 CS Utrecht, The Netherlands

e-mail: D.Schutter@uu.nl

\section{Introduction}

The view that the cerebellum is involved in the experience and regulation of emotions was already posited more than half a century ago $[1,2]$. One of the first papers reporting on cerebellar involvement in the experience of emotions involved a patient who underwent electrical stimulation of the dentate nucleus and superior peduncle and reported unpleasant feelings [3]. Furthermore, electrophysiological responses in several limbic structures, including the hippocampus, amygdala, and septum were recorded following electrical stimulation of the fastigial part of the deep cerebellar nuclei in mammals $[4,5]$. Additional support in humans was provided by an emotionally disturbed patient who received electrical stimulation of the fastigial nucleus [6]. It was found that the electrical discharges induced by electric stimulation correlated with the experience of anger and tension. Changing the focus from the deep cerebellar nuclei to the more superficial layers of the cerebellum Heath demonstrated that chronic stimulation of the vermis using implanted subdural electrodes normalizes behavior in severe emotionally dysregulated patients [7]. Schmahmann and Sherman were the first to describe a clinical condition, the cerebellar cognitive-affective syndrome characterized by executive dysfunctions, behavioral disinhibition and emotion dysregulation that resulted from posterior and vermal lesions of the cerebellum [8]. Similar behavioral problems have been observed in children with extensive lesions to the vermis [9]. Even though cerebellar lesions certainly do not always lead abnormal or pathological behavior, Parvizi and colleagues, for example, have provided evidence for cerebellar damage and deficits in the regulation of pathological crying and laughter [10]. Additional evidence for emotion dysregulation has been provided by findings showing cerebellar abnormalities in 
disorders that are characterized by emotion dysregulation such as attention deficit/hyperactivity disorder, bipolar disorder, schizophrenia, and depression [11-13]. The fact that the cerebellum is reciprocally connected to a broad range of limbic structures including the amygdala, hippocampus, and septum, as well as the cerebral cortex including the prefrontal areas [4, 14], provides a strong neuroanatomical argument in favor of cerebellar involvement in emotion regulation. Particularly, the connections between the cerebellum and limbic system and the cerebello-thalamo-cortical projections provide a neuroanatomical foundation for cognition and emotion interactions $[15,16]$. Taken together, there seems to be sufficient empirical ground to assume that the cerebellum plays a role in the regulation of emotions.

The critical insights for the function of the cerebellum still come from early invasive electric stimulation studies. A new noninvasive way of obtaining direct insights into the cerebellar function is transcranial magnetic stimulation (TMS). TMS utilizes magnetic fields to get electrical currents in the brain and influence neuronal activity. In a previous study, we applied high-frequency repetitive TMS (rTMS) over the medial cerebellum to facilitate cerebellar function and found elevations in mood and changes in frontal electric activity [17]. Interestingly, electrophysiological studies that addressed the functional relationships between the different frequency bandwidths of the human electroencephalogram (EEG) suggest that elevated ratios of slow and fast brain wave oscillations recorded over the frontal midline indicate impaired emotion regulation [18].

The aim of the present sham and occiput controlled within-subjects study was to obtain electrophysiological and phenomenological evidence for cerebellar involvement in emotion regulation by applying slow frequency inhibitory rTMS. It was hypothesized that $1 \mathrm{~Hz}$ rTMS over the medial cerebellum in healthy volunteers would (1) lead to elevations in frontal EEG ratios during emotion regulation, and (2) increases in negative mood as a result of impaired emotion regulation.

\section{Materials and Methods}

\section{Participants}

Twelve healthy nonsmoking female volunteers participated in the study. All participants were aged between 18 and 23 years, were right-handed, and used oral contraceptives. None of the subjects had a history of psychiatric or neurological conditions and had normal or corrected-tonormal vision. Only female subjects were included in the present study because research has shown that compared to males, females are more vulnerable to disorders associated with emotion dysregulation [19]. Written informed consent was obtained, and volunteers were paid for participation. The study was approved by the medical ethical committee of the Utrecht University in accordance with the declaration of Helsinki. All participants were unaware of the hypotheses being tested in the study.

\section{Transcranial Magnetic Stimulation}

Slow frequency rTMS was performed using a Neotonus (Atlanta) magnetic brain stimulator (maximum output 2,300 A peak/1750 VAC peak) with an iron core coil. Sham rTMS was performed using an identical coil, but with a metal plate built in the housing directly under the iron-core (Neotonus, Atlanta). The coil mimics the sound click and sensation of real TMS, but the brain is shielded from actual stimulation.

\section{Electroencephalogram}

EEG was recorded from 32 scalp locations according to the International 10-20 EEG System using Ag-AgCl-tipped electrodes (sampling rate: $256 \mathrm{~Hz}$ ). Electro-oculogram (EOG) was recorded from bipolar montages from the suband supraorbital regions of the right eye and the outer canthi of the eyes. Raw EEG recordings were made with the ActiveTwo system (BioSemi, Amsterdam, The Netherlands) relative to the common mode sense (CMS). By physically integrating the first amplifier stage with a sintered $\mathrm{Ag}-\mathrm{AgCl}$ electrode, extremely low-noise recordings free of interference can be achieved (www.biosemi.com).

\section{Emotion Regulation}

Exposure to unpleasant pictures from the International Affective Picture System (IAPS) can have short-term negative mood effects [20]. In the emotion regulation task (ERT), participants were presented with a series of unpleasant highly arousing pictures depicted from the IAPS $^{1}$ [21]. Prior to each block, subjects were instructed

\footnotetext{
${ }^{1}$ IAPS Picture numbers were as follows: Version 1-Unpleasant: $1050,1120,1301,3051,3053,3060,3064,3100,3102,3180,3350$, $6200,6211,6212,6243,6312,6313,6360,6370,6371,6830,7380$, 8480, 9040, 9042, 9050, 9405, 9433, 9571, 9921; Neutral: 2190, 7000, 7006, 7010, 7020, 7130, 7031, 7182, 7187, 7234, 7237, 7490, 7491, 7710, 7950. Version 2-Unpleasant: 1090, 1300, 2691, 2730, $3010,3062,3080,3120,3261,3400,3500,6210,6230,6350,6510$, 6530, 8230, 9006, 9160, 9250, 9252, 9410, 9500, 9600, 9611, 9800, 9810, 9910, 9911, 9920; Neutral: 2200, 7025, 7035, 7040, 7050, 7060, 7090, 7100, 7150, 7160, 7185, 7205, 7207, 7233, 7235. Version 3-Unpleasant: 1201, 3000, 3030, 3063, 3110, 3140, 3150, 3170, $3071,3350,3530,6244,6250,6260,6300,6410,6540,6550,6560$, 6570, 6821, 9120, 9300, 9400, 9420, 9570, 9620, 9621, 9622, 9630; Neutral: 2210, 5534, 7002, 7004, 7009, 7030, 7034, 7080, 7110, $7140,7170,7180,7217,7224,7500$.
} 
to either just experience any feelings elicited by the unpleasant pictures or to neutralize any feelings by cognitive re-appraisal. The task consisted of ten 1-min blocks containing three different stimulus presentations of 20 s. Prior to each block, the instruction "experience" or "re-appraise" appeared on the screen. An additional five blocks containing three low-arousing neutral stimuli were put into the task to avoid habituation effects. During the neutral blocks, subjects were instructed to simply look at the stimulus presentation. Blocks were randomized with the restriction that no block type would occur more than twice in succession, and the blocks were separated by a $1 \mathrm{~min}$ relaxation period. Three parallel versions were constructed from the IAPS set, which did not statistically differ on the unpleasant and arousal dimension. Pictures were presented on a 16 -in. computer screen at a distance of $1 \mathrm{~m}$ from the subject.

\section{Mood Assessment}

The shortened 32-item version of the Profile of Mood States (POMS) was used to index effects on negative mood defined as the mean score on the tension, anger, fatigue, and depression scales [22, 23]. Positive mood was assessed by the vigor scale of the POMS. It should be noted that even though the vigor scale is the only POMS scale related to positive mood, several lines of research have established that the vigor scale correlates high with feelings of wellbeing and positive emotions [24, 25]. Moreover, slight but systematic differences in mood state of normal subjects are not likely to be revealed by conventional ordinal response scales as used in the standard POMS. To enhance sensitivity, a visual analogue scale ranging from 0 (not at all) to 100 (extremely) was applied [26, 27]. Mood scores were acquired by calculating the sum of scores on the relevant items.

\section{Procedure}

In a single-blind, sham and occipital-controlled crossover design, participants received $20 \mathrm{~min}$ of $1 \mathrm{~Hz}$ subthreshold rTMS over (1) the medial cerebellum targeting the vermis, (2) midline occiput, (3) sham rTMS over the medial cerebellum (1,200 pulses $\mathrm{p} / \mathrm{session})$. Each rTMS session was separated by $24 \mathrm{~h}$ and day time controlled. Order of session was randomized and counterbalanced across the group.

With the inion serving as reference point [27, 28], the medial cerebellum targeting the vermis for real $(\mathrm{Cbl})$ and sham (Shm) rTMS was defined as the point located $1 \mathrm{~cm}$ below the inion [29]. The occiput (Occ) served as the second control site and was located $3 \mathrm{~cm}$ above the inion. Stimulation intensity was fixed at $45 \%$ of maximum machine output (for the rationale, see [30]). The modified figure-of-eight iron core coil generates relative little neck muscle contractions. Even though, there is no large difference between rTMS applied over the medial cerebellum and occiput, the latter condition was introduced to control for confound of neck muscle contractions. The current in the coil was directed downward which will induce an upward current in the target region and is assumed to be the most optimal position for cerebellar stimulation [31]. Stimulation parameters were in accordance with the safety guidelines as formulated by the International Federation of Clinical Neurophysiology (http:// www.ifcn.info).

Prior to the day of their first experimental sessions, on a separate day, participants were invited to the laboratory where safety issues, experimental procedures were explained to the subject, and informed consent was obtained. A safetyscreening list was administered to check for contraindications [32], and right-handedness (mean $\pm \mathrm{SD}, 44 \pm 3.0$ ) was established with the Edinburgh Handedness Inventory [33]. Individual motor threshold (MT) from the left primary motor cortex using the thumb movement visualization method was determined (mean $\pm \mathrm{SD}, 54.9 \pm 8.6$ ) [34]. On experimental days, participants were asked to refrain from taking psychotropic substances, including alcohol, coffee, tea, and chocolate 2-h prior to experimentation. The POMS was administered prior and immediately after rTMS. After the second POMS administration, the participants performed the ERT. Electric brain activity was recorded continuously during the neutral, re-appraise, and experience conditions of the ERT. Each session was concluded by a third POMS.

\section{Data Reduction and Analyses}

Off-line raw EEG signals were re-referenced to the $\mathrm{Cz}$ electrode, digitally band-pass filtered (1-30 Hz: $24 \mathrm{~dB} /$ octave) chunked into 4-s epochs and corrected for horizontal and vertical eye movements using the Gratton and Coles method (BrainVision, Munich). EEG epochs containing residual muscle movements and other artefacts resulting in amplitudes greater than $\pm 50 \mu \mathrm{V}$ were rejected prior to further analysis. The designation of an artefact in one of the leads resulted in removal of that epoch for all channels in order to ensure that the remaining data were identical in time (Fig. 1). For each 4-s epochs, a fast Fourier transform method (Hamming window length: 10\%) was applied to obtain estimates of spectral power $\left(\mu \mathrm{V}^{2}\right)$ of slow wave (delta, 1-3 Hz; theta, 4-7 Hz) and fast wave (beta, 13-30 Hz) brain oscillations. Delta-beta and theta-beta EEG ratios were calculated for the frontal midline electrode for the three conditions. Emotion regulation was indexed by calculating difference scores of the EEG ratios recorded during the experience and re-appraise condition. Changes in negative mood were investigated by comparing the baseline corrected scores prior and after the emotion regulation test between the rTMS conditions. Due to technical difficulties, 


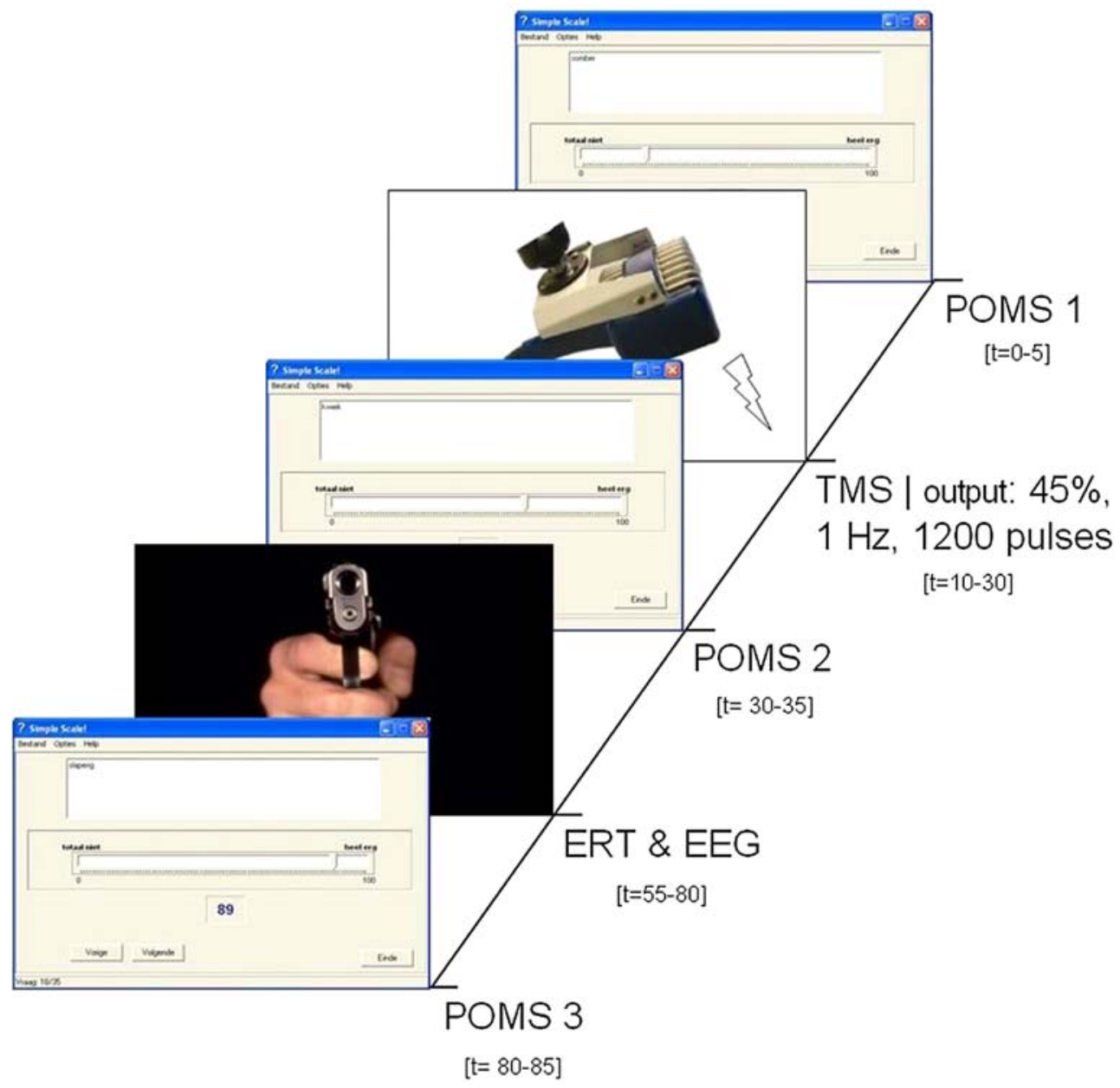

Fig. 1. Timeline of the experimental procedure. $t$ time in minutes

data for one subject were lost, and this subject was excluded from further analyses. Nonparametric statistical analyses were performed. The small sample size required nonparametric statistical testing as these are less susceptible to outlier confounds and thus more conservative. Furthermore, nonparametric tests also do not make a priori assumptions about the distribution of the values $[35,36]$. The alpha level of significance was set at $p<0.05$.

\section{Results}

TMS was well-tolerated by all participants and no adverse events occurred. In spite of the fact that a number of participants reported differences in felt sensation between sessions, all had been in the supposition of receiving real rTMS in the three sessions. This shows that although the sham condition may not have been perfect, blinding was nevertheless effective.

A Friedman test for related samples showed no acute changes in negative $\left(\chi_{\mathrm{F}}^{2}(2,9)=0.42, p=0.81\right.$, two-tailed) and positive $\operatorname{mood}\left(\chi_{\mathrm{F}}^{2}(2,9)=3.62, p=0.16\right.$, two-tailed $)$ were present after $20 \mathrm{~min}$ of $1 \mathrm{~Hz}$ rTMS. In the ERT, no significant effects of rTMS on emotion regulation as indexed by changes in delta-beta $\left(\chi_{\mathrm{F}}^{2}(2,9)=1.27, p=0.53\right.$, twotailed) and theta-beta EEG ratio difference scores were observed $\left(\chi_{\mathrm{F}}^{2}(2,9)=0.55, p=0.76\right.$, two-tailed). However, a significant condition effect of rTMS on negative mood was observed after the ERT $\left(\chi_{\mathrm{F}}^{2}(2,9)=9.46, p=0.01\right.$, twotailed). Hypotheses-driven post hoc Wilcoxon signed ranks tests (one-tailed) revealed significant increases of negative mood in the cerebellar versus sham rTMS condition $(z=-2.04, p=0.01$, effect size $r, 0.62)$, and a significant increase of negative mood in the cerebellar compared to occipital rTMS $(z=-1.87 ; p=0.03$, effect size $r, 0.56)$. Importantly, the sham-occipital rTMS comparison was not significant $(z=-0.67: p=0.25)$. Finally, no TMS-related changes in positive mood after the ERT were found $\left(\chi_{\mathrm{F}}^{2}(2,9)=0.72, p=0.7\right.$, two-tailed $)$. Figure 2 displays the average scores for the baseline-corrected differences of negative mood before and after the ERT for each rTMS condition. 


\section{Discussion}

The results of the present study add to the growing recognition that the cerebellum is implicated in cognitive processes associated with emotion control [37].

The first theoretical notions which suggested that the cerebellum has a regulatory role in intellectual and emotional functioning originated from animal studies. In addition, to the pioneering work of Heath and colleagues, Cooper and colleagues documented spontaneous mood changes and emotional experiences of subjects who received electrical cerebellar stimulation to treat epilepsy [38]. Additionally, chronic cortical cerebellar stimulation resulted in unexpected positive side effects that included improvements of aggression and anxiety [39]. In agreement, more recent findings have shown that the success of pharmacological and psychological interventions in the treatment of these disorders is associated with improvements in cerebellar function [11].

Given its homogenous morphology, it has been proposed that the cerebellum is part of a cortical-thalamic-cerebellocortical (CTCC) circuit that, analogous to the traditional view of the cerebellar modulatory properties in motor activity, monitors and regulates mental states [40]. The notion of the cerebellum as a universal regulator is further supported by studies showing cerebellar involvement in timing and integration of somatic and visceral information $[41,42]$. Observations of crossed cerebellar hypoperfusion after cortical stroke and crossed cerebello-cerebral diaschisis in patients with cerebellar stroke give further evidence for the CTCC circuit. This may have implications for the cerebello-cerebral interactions in emotion regulation, particularly since emotion regulation is considered a function of the frontal cortex [43, 44]. Daskalakis and colleagues applied paired-pulse TMS and showed reduced cerebello- thalamo-cortical inhibition in schizophrenic patients, providing the first direct evidence for a link between behavioral dysregulation and disturbed communication between the cerebellum and frontal cortex [45]. Although the present results do not allow us to draw strong inferences on the neurophysiological mechanisms involved in this study, we aimed to inhibit neuronal excitability of the superficial layers of the cerebellar vermis. There is some indirect evidence showing that lesions to the anterior cerebellar vermis can reduce mesolimbic dopamine activity [46]. This may have set the stage for increased susceptibility to negative-laden stimuli on the subcortical level. Interestingly, reductions in dopaminergic activity in the mesolimbic system has been linked to depression [47]. Together with the notion that the cerebellum is implicated in cognitive processes associated with calibrating internal and external information processing streams as well as with affect regulation $[8,41]$, it is conceivable that the combination of disrupted cerebellar function and the increased susceptibility to negative laden stimuli may have resulted in the increases in consciously-experienced negative mood.

Unfortunately, in the present study no evidence was found for changes in electro-cortical activity over the frontal midline during emotion regulation following cerebellar rTMS. One of the possible reasons for this absence may be found in a recent meta-analysis which suggests that EEG ratios reflect stable trait-like properties and are less sensitive to state-related changes [48]. Clearly, more research is necessary to elucidate the exact contributions of state and trait properties of electric brain activity to the EEG ratios and the assumed relation with emotion regulation.

Even though recent findings show that physiological responses can be obtained at stimulation intensities well below the motor threshold [17, 49], higher intensities than
Fig. 2. Mean and SE of the means of the baseline corrected scores on negative mood before (left panel) and after the emotion regulation task (right panel). $\mathrm{Cbl}$ cerebellum, Occ occiput, Shm sham
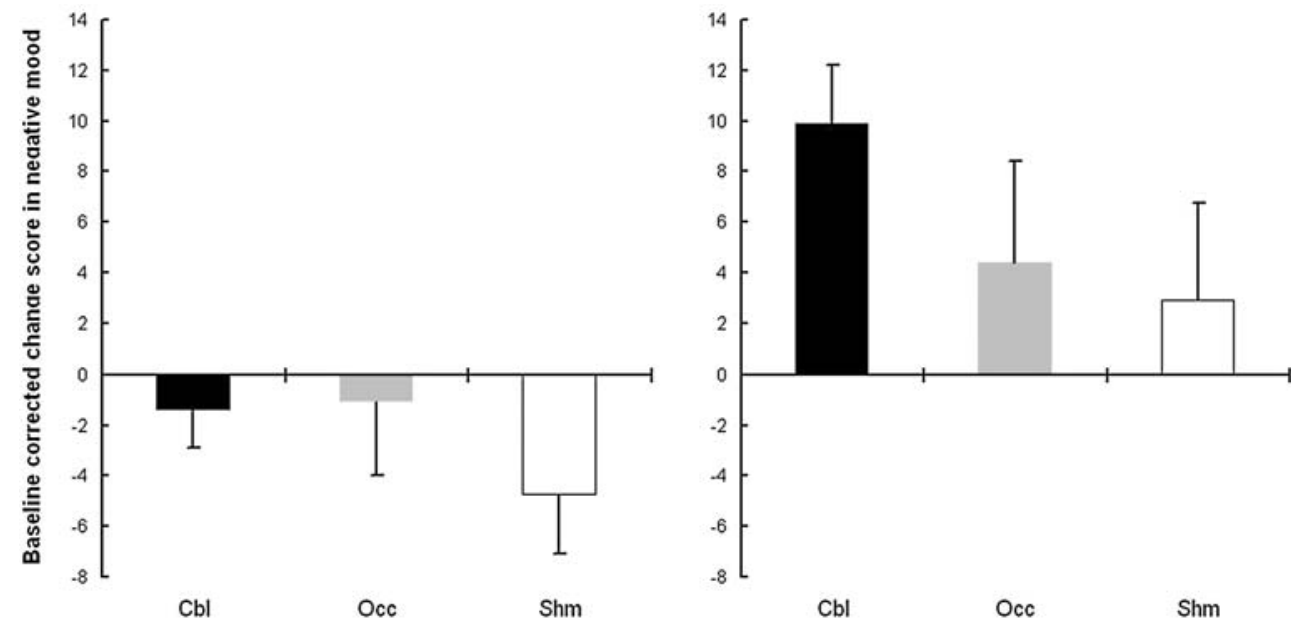

TMS condition 
the currently applied stimulation of $45 \%$ machine output which corresponds to $\sim 80 \%$ MT in the present sample, may have had more pronounced effects on mood and EEG ratios. However, findings from a recent study show that intensities as low as $60 \%$ MT are sufficient enough to evoke EEG responses in superficial nerve tissue of the primary motor cortex, suggesting that intensities well below the neural excitation threshold can have significant physiological effects [49].

The present design did not detect mood changes immediately after cerebellar rTMS or reliable increases in EEG ratios during emotion regulation. Despite the withinsubjects design and hypothesis-driven nonparametric testing, the study might have been underpowered due to the relative small sample size increasing the likelihood of a Type II error. It should however be noted that subthreshold slow frequency rTMS does usually not result in self-reported changes in mood [50-51], which makes the elevations in negative mood after emotion regulation following cerebellar rTMS even more noteworthy. Furthermore, slow frequency rTMS to the medial cerebellum may have introduced a state of heightened "physiological" susceptibility that may have contributed to the increases in negative mood after the emotional challenge. Moreover, these findings add to the proposal that a single session of slow frequency inhibitory rTMS is able to transiently alter the organism's motivational state without causing immediate effects on mood [51], and holds with the idea that rTMS combined with a supplementary challenge is critical for eliciting effects on consciously experienced mood [52].

Concerning the site of stimulation, spatial resolution was controlled for by including an adjacent occipital rTMS condition in the experimental design to dissociate cerebellarfrom cortical-related effects. Additionally, inspection of a structural brain scan acquired in a previous study confirmed that rTMS applied $1 \mathrm{~cm}$ below the inion indeed targets the medial cerebellum [28]. Nonetheless, despite the midline target sites, the low stimulation intensity and focality of the iron core coil, the possibility that as a result of focality loss in the posterior fossa peripheral nerves were also stimulated cannot be completely ruled out.

\section{Conclusion}

The ability to regulate one's emotions and feelings is vital for psychological well-being [53], and various forms of psychopathology can be understood in terms of emotion dysregulation. In the current study, slow frequency rTMS was applied to inhibit cerebellar activity, and evidence for reduced emotion regulation was found. These effects are in agreement with our earlier observations in which we aimed to facilitate rather than inhibit cerebellar function. In conclusion, the results of this study with healthy volunteers provide the first direct evidence for cerebellar involvement in emotion regulation and add to the growing recognition that the cerebellum is implicated in processes associated with the control of mood and emotion [54].

Acknowledgements This work was supported by an Innovational Research Grant (VENI 451-04-070) from the Netherlands Organization for Scientific Research (NWO). We would like to thank the anonymous reviewers for their valuable comments.

Open Access This article is distributed under the terms of the Creative Commons Attribution Noncommercial License which permits any noncommercial use, distribution, and reproduction in any medium, provided the original author(s) and source are credited.

\section{References}

1. Snider RS (1945) A tectocerebellar pathway. Anat Rec 91:299

2. Anand BK, Malhorta CL, Singh B, Dua S (1959) Cerebellar projections to the limbic system. J Neurophysiol 22:451-458

3. Nashold BS, Slaughter DG (1969) Effects of stimulating or destroying the deep cerebellar regions in man. J Neurosurg $31: 172-186$

4. Snider RS, Maiti A (1976) Cerebellar contributions to the Papez circuit. J Neurosci Res 62:133-146

5. Heath RG, Dempsey CW, Fontana CJ, Myers WA (1978) Cerebellar stimulation: effects on septal region, hippocampus and amygdala of cats and rats. Biol Psychiatry 13:501-529

6. Heath RG, Cox AW, Lustick LS (1974) Brain activity during emotional states. Am. J Psychiatry 131:858-862

7. Heath RG (1977) Modulation of emotion with a brain pacemaker: treatment for intractable psychiatric illness. J Nerv Ment Dis 165:300-317

8. Schmahmann JD, Sherman JC (1998) The cerebellar cognitive affective syndrome. Brain 121:561-579

9. Levisohn L, Cronin-Golomb A, Schmahmann JD (2000) Neuropsychological consequences of cerebellar tumour resection in children: cerebellar cognitive affective syndrome in a paediatric population. Brain 123:1041-1050

10. Parvizi J, Anderson SW, Martin CO, Damasio H, Damasio AR (2001) Pathological laughter and crying: a link to the cerebellum. Brain 124:1708-1719

11. Konarski JZ, McIntyre RS, Grupp LA, Kennedy SH (2005) Is the cerebellum relevant in the circuitry of neuropsychiatric disorders? J Psychiatry Neurosci 30:178-186

12. Berquin PC, Giedd JN, Jacobsen LK, Hamburger SD, Krain AL, Rapoport JL et al (1998) The cerebellum in attention-deficit/ hyperactivity disorder: a morphometric study. Neurology 50:1087-1093

13. Starkstein SE, Robinson RG, Berthier ML, Price TR (1988) Depressive disorders following posterior circulation as compared with middle cerebral artery infarcts. Brain 111:375-387

14. Middleton FA, Strick PL (2001) Cerebellar projections to the prefrontal cortex of the primate. J Neurosci 21:700-712

15. Manto MU (2006) On the cerebello-cerebral interactions. Cerebellum 5:286-288

16. Schutter DJLG, van Honk J (2006) An electrophysiological link between the cerebellum, cognition and emotion: Frontal theta EEG activity to single pulse cerebellar TMS. Neuroimage 33:1227-1231

17. Schutter DJLG, van Honk J, d, 'Alfonso AAL, Peper JS, Panksepp J (2003) High frequency repetitive transcranial magnetic over the medial cerebellum induces a shift in the prefrontal 
electroencephalography gamma spectrum: a pilot study in humans. Neurosci Lett 336:73-76

18. Hobbs MJ, Clarke AR, Barry RJ, McCarthy R, Selikowitz M (2007) EEG abnormalities in adolescent males with AD/HD. Clin Neurophysiol 118:363-371

19. Weissman MM, Bland R, Joyce PR, Newman S, Wells JE, Wittchen HU (1993) Sex differences in rates of depression: crossnational perspectives. J Affect Disord 29:77-84

20. Smith JC, Bradley MM, Lang PJ (2005) State anxiety and affective physiology: effects of sustained exposure to affective pictures. Biol Psychol 69:247-260

21. Lang PJ, Bradley MM, Cuthbert BN (1999) International Affective Picture System (IAPS): Instruction manual and affective ratings. Technical Report A-4, Center for Research in Psychophysiology. University of Florida, Gainesville

22. Shacham S (1983) A shortened version of the Profile of Mood States. J Pers Assess 47:305-306

23. van Honk J, Tuiten A, Verbaten R, van den Hout M, Koppeschaar H, Thijssen J et al (1999) Correlations among salivary testosterone, mood, and selective attention to threat in humans. Horm Behav 36:17-24

24. Lee KH, Egleston PN, Brown WH, Gregory AN, Barker AT, Woodruff PW (2003) A simple self-report diary for assessing psychosexual function in hypogonadal men. J Androl 24:688-698

25. Horowitz M, Adler N, Kegeles S (1988) A scale for measuring the occurrence of positive states of mind: a preliminary report. Psychosom Med 50:477-483

26. Bond A, Lader M (1974) The use of analogue scales in rating subjective feelings. Br J Med Psychol 47:211-218

27. Schutter DJLG, Kammers MPM, Enter D, van Honk J (2006) A case of illusory own-body perception after transcranial magnetic stimulation over the cerebellum. Cerebellum 5:238-240

28. Torriero S, Oliveri M, Koch G, Caltagirone C, Petrosini L (2004) Interference of left and right cerebellar rTMS with procedural learning. J Cogn Neurosci 16:1605-1611

29. Lee KH, Egleston PN, Brown WH, Gregory AN, Barker AT, Woodruff PW (2007) The role of the cerebellum in subsecond time perception: evidence from repetitive transcranial magnetic stimulation. J Cogn Neurosci 19:147-157

30. Epstein CM, Davey KR (2002) Iron-core coils for transcranial magnetic stimulation. J Clin Neurophysiol 19:376-381

31. Ugawa Y, Uesaka Y, Terao Y, Hanajima R, Kanazawa I (1995) Magnetic stimulation over the cerebellum in humans. Ann Neurol 37:703-713

32. Keel JC, Smith MJ, Wassermann EM (2000) A safety screening questionnaire for transcranial magnetic stimulation. Clin Neurophysiol 112:720

33. Oldfield RC (1971) The assessment and analysis of handedness: the Edinburgh inventory. Neuropsychologia 9:97-113

34. Schutter DJLG, van Honk J (2006b) A standardized motor threshold estimation procedure for transcranial magnetic stimulation research. J ECT 22:176-178

35. Siegel S, Castellan NJ (1988) Non-parametric statistics for the behavioural sciences. McGraw-Hill, New York, NY

36. Clark-Carter D (1997) Doing quantitative psychological research. Psychology Press, East Sussex
37. Schmahmann JD, Caplan D (2006) Cognition, emotion and the cerebellum. Brain 129:290-292

38. Cooper IS, Upton ARM (1985) Therapeutic implications of modulation of metabolism and functional activity of cerebral cortex by chronic stimulation of cerebellum and thalamus. Biol Psychiatry 20:809-811

39. Riklan M, Marisak I, Cooper IS (1974) Psychological studies of chronic cerebellar stimulation in man. In: Cooper IS, Riklan M, Snider RS (eds) The cerebellum, epilepsy and behavior. Plenum, New York, pp 285-342

40. Andreasen NC (1999) A unitary model of schizophrenia: Bleuler's "fragmented phrene" as schizencephaly. Arch Gen Psychiatry 56:781-787

41. Ivry RB, Spencer RM (2004) The neural representation of time. Curr Opin Neurobiol 14:225-232

42. Zhu JN, Yung WH, Kwok-Chong Chow B, Chan YS, Wang JJ (2006) The cerebellar hypothalamic circuits: Potential pathways underlying cerebellar involvement in somatic-visceral integration. Brain Res Rev 52:93-106

43. Kamouchi M, Fujishima M, Saku Y, Ibayashi S, Iida M (2004) Crossed cerebellar hypoperfusion in hyperacute ischemic stroke. J Neurol Sci 225:65-69

44. Komaba Y, Osono E, Kitamura S, Katayama Y (2000) Crossed cerebellocerebral diaschisis in patients with cerebellar stroke. Acta Neurol Scand 101:8-12

45. Daskalakis ZJ, Christensen BK, Fitzgerald PB, Fountain SI, Chen R (2005) Reduced cerebellar inhibition in schizophrenia: a preliminary study. Am J Psychiatry 162:1203-1205

46. Albert TJ, Dempsey CW, Sorenson CA (1985) Anterior cerebellar vermal stimulation: Effect on behaviour and basal forebrain neurochemistry in rat. Biol Psychiatry 20:1267-1276

47. Nestler EJ, Carlezon WA Jr (2006) The mesolimbic dopamine reward circuit in depression. Biol Psychiatry 59:1151-1159

48. Snyder SM, Hall JR (2006) A meta-analysis of quantitative EEG power associated with attention-deficit hyperactivity disorder. J Clin Neurophysiol 23:440-455

49. Komssi S, Savolainen P, Heiskala J, Kähkönen S (2007) Excitation threshold of the motor cortex estimated with transcranial magnetic stimulation electroencephalography. Neuroreport 18:13-6

50. d'Alfonso AAL, van Honk J, Hermans E, Postma A, de Haan EHF (2000) Laterality effects in selective attention to threat after repetitive transcranial magnetic stimulation at the prefrontal cortex in female subjects. Neurosci Lett 280:195-198

51. Schutter DJLG, van Honk J (2006) Increased positive emotional memory after repetitive transcranial magnetic stimulation over the orbitofrontal cortex. J Psychiatry Neurosci 31:101-104

52. Rossini D, Magri L, Lucca A, Giordani S, Smeraldi E, Zanardi R (2005) Does rTMS hasten the response to escitalopram, sertraline, or venlafaxine in patients with major depressive disorder? A double-blind, randomized, sham-controlled trial. J Clin Psychiatry 66:1569-1575

53. Ochsner KN, Gross JJ (2005) The cognitive control of emotion. Trends Cogn Sci 9:242-249

54. Schutter DJLG, van Honk J (2005) The cerebellum on the rise in human emotion. Cerebellum 4:290-294 